\title{
Equivalent Circuit Model of an Optomechanical MEMS Electric Field Strength Sensor ${ }^{+}$
}

\author{
Andreas Kainz ${ }^{1, *}\left(\mathbb{D}\right.$, Wilfried Hortschitz ${ }^{2}$, Harald Steiner ${ }^{2}$, Michael Stifter ${ }^{2}$ and \\ Franz Keplinger ${ }^{1}$ \\ 1 Institute of Sensor and Actuator Systems, TU Wien, 1040 Vienna, Austria; franz.keplinger@tuwien.ac.at \\ 2 Department for Integrated Sensor Systems, Danube University Krems, 2700 Wiener Neustadt, Austria; \\ wilfried.hortschitz@donau-uni.ac.at (W.H.); harald.steiner@donau-uni.ac.at (H.S.); \\ michael.stifter@donau-uni.ac.at (M.S.) \\ * Correspondence: andreas.kainz@tuwien.ac.at; Tel.: +43-1-58801-76697 \\ + Presented at the Eurosensors 2018 Conference, Graz, Austria, 9-12 September 2018.
}

Published: 3 December 2018

\begin{abstract}
We present a simple equivalent circuit model for the transfer function of an optomechanical MEMS transducer capable of distortion-free electric field strength measurements. This model allows not only to qualitatively understand the characteristics of the transducer but also takes into account parasitic effects and material properties. Such parasitic effects have been observed while evaluating the first results of electric field measurements performed with the sensor. The model helped to identify and diminish these parasitic effects.
\end{abstract}

Keywords: MEMS; sensors; electric field sensor; electric field; modelling; equivalent circuit models

\section{Introduction}

Monitoring the strength of static and quasistatic electric fields (E-field) is key for many scientific and industrial purposes, e.g., improving the reliability of weather forecast, researching charge distributions in cloud systems or safety in the vicinity of high-voltage infrastructure. Despite this high demand, as of yet, there exist no state-of-the-art sensors which are sufficiently capable of fulfilling this task. This is either due to the necessity for grounded components which severely distort the E-field (field mills [1,2]), large temperature dependence (electrooptical sensors [3,4]) or limited bandwidth (antennae [5,6]). Recently, a new transduction scheme for the electric field strength was presented which does not suffer from these drawbacks [7].

This transduction exploits the electrostatic induction occurring in conducting bodies inside an electric field $E_{0}$. The surface charges arising due to the induction are themselves subject to $E_{0}$ leading to a force $F_{\text {es }}$ proportional to $E_{0}^{2}$. The MEMS sensors are designed such that the electric field is concentrated across a narrow gap separating a moveable silicon mass from a fixed Si part (Figure 1a), which increases $F_{\text {es. }}$. The force leads to a displacement of the Si mass which is read out optically.

This is achieved by light flux modulation with an optical shutter (Figure 1b). The optical shutter is composed of a $\mathrm{Cr}$ grid deposited on a glass chip and an identical grid etched into the moving mass of the Si chip. Thus, light which is introduced onto the top side of the sensor chip is modulated by the mass displacement caused by the electric field. This kind of readout has proven to be highly sensitive $[8,9]$.

Since the E-feld transduction scheme is new, little is known about its properties. Making use of an equivalent circuit model helps understanding a few basic characteristics and provides a qualitative understanding. 


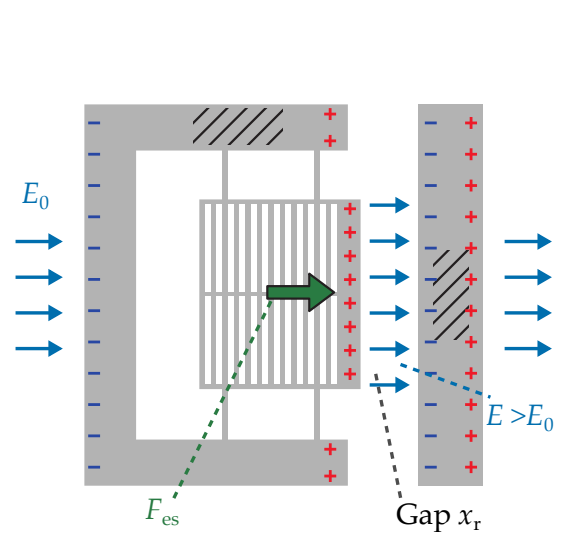

(a)

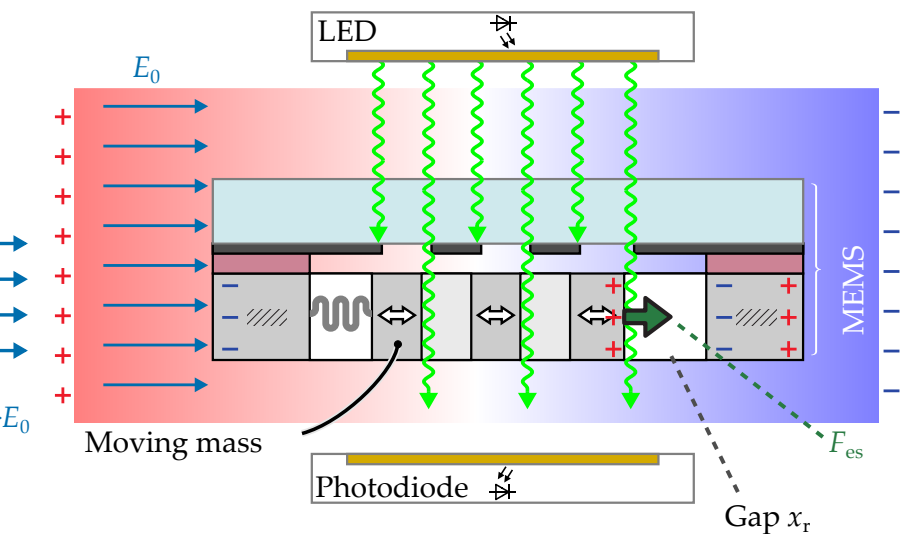

(b)

Figure 1. Schematic of the E-field transduction principle. (a) The electric field $E_{0}$ causes a polarisation in the two silicon domains separated by a small gap (width $x_{\mathrm{r}}$ ). The resulting surface charges are subject to $E_{0}$ and thus experience a force $F_{\mathrm{es}}$. Since the gap is very small compared to the dimensions of the silicon domain, the electric field $E$ inside the gap is larger than $E_{0}$, increasing $F_{\mathrm{es}}$ at the same time. (b) The force pulls the moving mass into the gap. This causes the optical shutter consisting of a Cr layer on the top glass chip and the holes inside the Si mass to change the transmitted light intensity.

The changing light flux is detected with a photodiode.

\section{Model}

The equivalent circuit model of the sensor's frequency-dependent transfer function $H_{\mathrm{s}}(\omega)$ presented here encompasses not only the sensor chip but also parts of the measurement setup. These parts are the capacitor plates used to provide the electric field to the MEMS. As depicted in Figure 2, the field plates of the experimental setup and the MEMS chip can be understood as three capacitors connected in series.

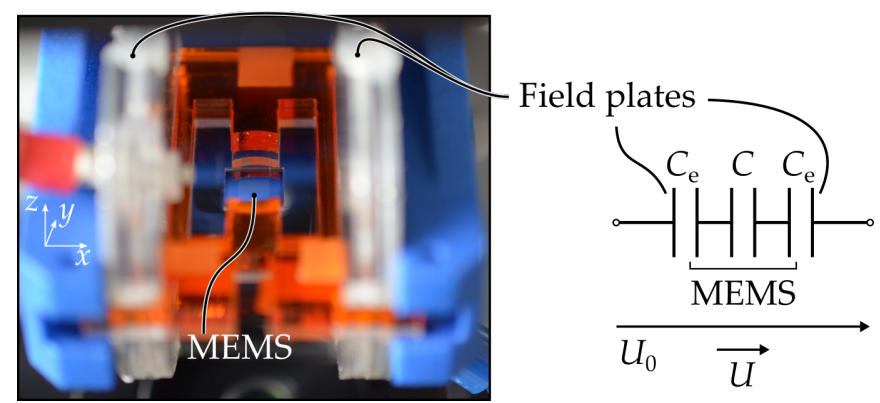

Figure 2. Equivalent circuit diagram of the E-field measurement setup. The ideal system can be understood as three capacitors connected in series and the electrostatic force exerted on the sensor as proportional to the square of the voltage drop at the centre capacitor $C$ which corresponds to the sensing gap of the MEMS.

The first capacitor comprises one of the aluminum plates and the opposing side face of the device layer of the MEMS chip. The second capacitor $C$ corresponds to the sensing gap of the MEMS chip and the third capacitor encompasses the remaining aluminum plate and opposing face of the MEMS chip. Since the chip is placed in the centre of the setup and the dimensions of the plates are the same, it follows with sufficient accuracy that the external capacitances are the same, i.e., $C_{e}$. The voltage $U_{0}$ applied to this voltage divider corresponds to the voltage which generates the electric feld $E_{0}=U_{0} / d_{\mathrm{p}}$ to be measured, where $d_{\mathrm{p}}$ is the distance between the field plates. The force picked up by the sensor corresponds to the square of the voltage drop $U$ at $C$, i.e., $F_{\text {es }} \propto U^{2}=H_{0}^{2} U_{0}^{2}$, where 
$H_{0}$ is the characteristic of the capacitive voltage divider which can be calculated as the fraction of the impedance of the sensing gap $Z_{0}$ over the total impedance $Z_{\text {tot}}$,

$$
H_{0}=\frac{Z_{0}}{Z_{\text {tot }}}=\frac{1}{1+2 \frac{C}{C_{\mathrm{e}}}}, \quad \text { with } \quad Z_{\text {tot }}=Z_{0}+2 Z_{\mathrm{e}}, \quad \text { and } \quad Z_{0, \mathrm{e}}=\frac{1}{\mathrm{i} \omega C_{0, \mathrm{e}}} .
$$

This expression is independent from $\omega$ and, thus, constant. Equation (1) corresponds to the ideal case, i.e., perfect conductance of the $\mathrm{Si}$ and infinite resistance of the gap between. Furthermore, it reveals that a low ratio $C / C_{\mathrm{e}}$ is in favour of a large voltage drop across $C$ and, thus, a large force $F_{\mathrm{es}}$.

This approach can also be used to explore what happens, if a parasitic resistance $R_{\mathrm{p}}$ in parallel to $C$ occurs. The impedance of a resistor in parallel with a capacitor is given as $Z=$ $R_{\mathrm{p}} /\left(\mathrm{i} \omega R_{\mathrm{p}} C+1\right)$. The corresponding voltage drop over the sensing capacitor is, therefore, proportional to the characteristic

$$
H(\omega)=\frac{Z}{Z_{\text {tot }}}=\frac{1}{\left(1+2 \frac{C}{C_{\mathrm{e}}}\right)\left(1+\frac{2}{\mathrm{i} \omega R_{\mathrm{p}}\left(C_{\mathrm{e}}+2 \mathrm{C}\right)}\right)}=\frac{H_{0}}{1+\frac{\omega_{\mathrm{c}}}{\omega}} .
$$

In contrast to the ideal case Equation (1), this expression is frequency dependent. It corresponds to a first order high-pass with corner frequency $\omega_{\mathrm{c}}=2 / R_{\mathrm{p}}\left(C_{\mathrm{e}}+2 C\right)$.

It can be seen that a finite parasitic resistance severely affects the E-feld transduction for low frequencies and DC. In particular, the bandwidth becomes clipped for frequencies $\omega<\omega_{\mathrm{c}}$. Note that $H(\omega)$ enters the transfer function quadratically, $A(\omega) \propto H^{2}(\omega) \chi(2 \omega)$, where $\chi$ corresponds to the mechanical characteristic of the MEMS [7,10]. Therefore, the linear increase of $|H(\omega)|$ reflects in a quadratic increase in $|A(\omega)|$ worsening the circumstance (see Figure 3a).

\section{Results}

The above described parasitic effect was encountered during the first measurements with the sensor. The model helped to identify its source as the plastic chip holder which introduced a resistance across the sensing gap (see Figure $3 b$ ).

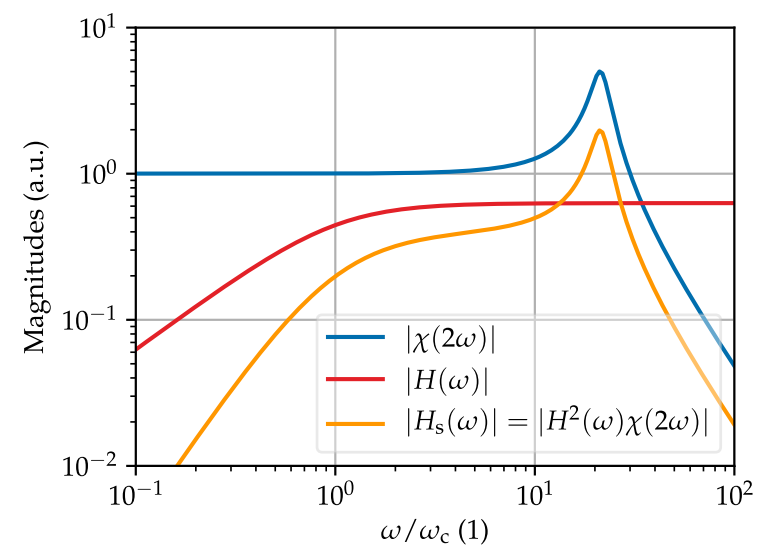

(a)

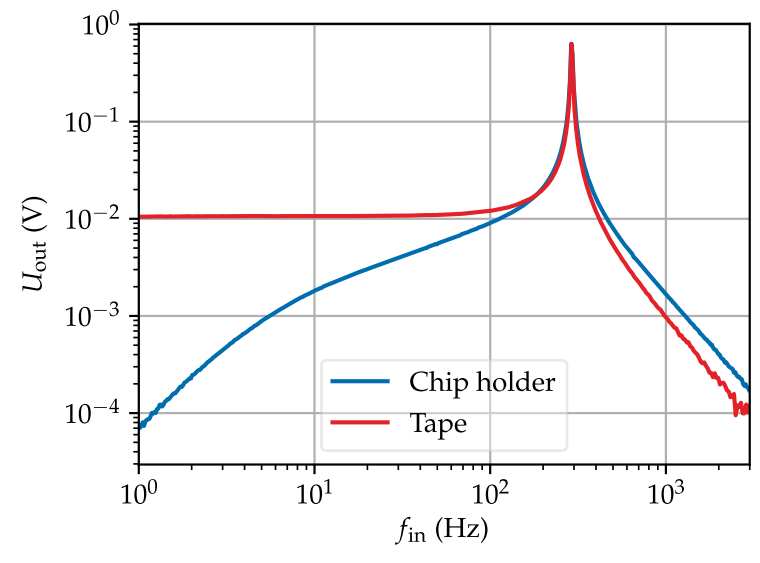

(b)

Figure 3. (a) Frequency dependent magnitudes of the functions $\chi(2 \omega)$ (blue), $H(\omega)$ (red) and $H^{2}(\omega) \chi(2 \omega) \propto A(\omega)$ (orange). It can be seen that the parasitic resistance significantly reduces the bandwidth of the E-field sensor. (b) E-field recordings of a MEMS sensor with $E_{0}=21 \mathrm{kV} / \mathrm{m}$. The measurement performed with the chip holder (blue) exhibits the bandwidth reduction as described by Equation (2). Replacing the chip holder with an adhesive tape solved this issue (red).

Estimating the capacitances $C$ and $C_{\mathrm{e}}$ from the geometry of MEMS and field plates and the corner frequency $f_{\mathrm{c}} \sim 10 \mathrm{~Hz}$ from the measured data, it was possible to roughly quantify the value of $R_{\mathrm{p}}$. The distance between the plate and the chip face is given by $d_{\mathrm{e}}=6.5 \mathrm{~mm}$. The mean area of the 
external capacitor can be estimated by $A_{\mathrm{e}}=3.64 \mathrm{~cm}^{2}$. Therefore, the external capacitance roughly has a value of $C_{\mathrm{e}} \approx \varepsilon_{0} A_{\mathrm{e}} / d_{\mathrm{e}}=0.49 \mathrm{pF}$.

The capacitance $C$ of the sensing gap can be estimated in a similar manner and has around $C \sim 0.13 \mathrm{pF}$ for a $10 \mu \mathrm{m}$ gap. This yields a parasitic resistance of $R_{\mathrm{p}}=1 / \pi f_{\mathrm{c}}\left(C_{\mathrm{e}}+2 C\right) \approx 45 \mathrm{G} \Omega$. Even though this value is quite large, the effect was clearly noticeable. Therefore, the choice of mounting the MEMS chip is crucial for proper measurements.

\section{Conclusions}

A very simple equivalent circuit model of an optomechanical MEMS electric field sensor was presented. The model also encompasses the effects of a parasitic resistor. This way it was possible to explain and identify the source of the reduced sensitivity of the sensor at low frequencies. The model can be expanded easily to account for, e.g., the finite condictance of Si.

Acknowledgments: This work was supported by the country of Lower Austria (NÖ, the European Regional Development Fund (ERDF) and the Austrian Science Fund (FWF project number P 28404-NBL).

Conflicts of Interest: The authors declare no conflict of interest. The founding sponsors had no role in the design of the study; in the collection, analyses, or interpretation of data; in the writing of the manuscript, and in the decision to publish the results.

\section{References}

1. Hill, D.A.; Kanda, M. The Measurement, Instrumentation, and Sensors Handbook XXV, Section 47, Electric Field Strength; CRC Press LLC: Boca Raton, FL, USA; IEEE Press: Piscataway, NJ, USA, 1999.

2. Kirkham, H. On the measurement of stationary electric fields in air. In Proceedings of the Conference on Precision Electromagnetic Measurements, Ottawa, ON, Canada, 16-21 June 2002.

3. Chmielak, B.; Waldow, M.; Matheisen, C.; Ripperda, C.; Bolten, J.; Wahlbrink, T.; Nagel, M.; Merget, F.; Kurz, H. Pockels effect based fully integrated, strained silicon electro-optic modulator. Opt. Express 2011, 19, 17212-17219.

4. Perry, D.; Chadderdon, S.; Gibson, R.; Shreeve, B.; Selfridge, R.H.; Schultz, S.M.; Wang, W.C.; Forber, R.; Luo, J. Electro-optic polymer electric field sensor. In Proceedings of the SPIE Smart Structures and Materials+ Nondestructive Evaluation and Health Monitoring, San Diego, CA, USA, 6-10 March 2011; International Society for Optics and Photonics: Bellingham, WA, USA, 2011; p. 79820Q.

5. Roussy, G.; Agbossou, K.; Dichtel, B. Vector electric field measurement using a non-interfering sensor. Meas. Sci. Technol. 2000, 11, 1145.

6. Chiang, F.C.; Pribyl, P.; Gekelman, W.; Lefebvre, B.; Chen, L.J.; Judy, J.W. Microfabricated flexible electrodes for multiaxis sensing in the large plasma device at UCLA. IEEE Trans. Plasma Sci. 2011, 39, 1507-1515.

7. Kainz, A.; Steiner, H.; Schalko, J.; Jachimowicz, A.; Kohl, F.; Stifter, M.; Beigelbeck, R.; Keplinger, F.; Hortschitz, W. Distortion-free measurement of electric field strength with a MEMS sensor. Nat. Electron. 2018, 1, 68 .

8. Hortschitz, W.; Steiner, H.; Stifter, M.; Kohl, F.; Kahr, M.; Kainz, A.; Raffelsberger, T.; Keplinger, F. Novel high resolution MOEMS inclination sensor. In Proceedings of the IEEE SENSORS 2014 Proceedings, Valencia, Spain, 2-5 November 2014; pp. 1893-1896, doi:10.1109/ICSENS.2014.6985399.

9. Middlemiss, R.; Samarelli, A.; Paul, D.; Hough, J.; Rowan, S.; Hammond, G. Measurement of the Earth tides with a MEMS gravimeter. Nature 2016, 531, 614-617.

10. Kainz, A.; Hortschitz, W.; Steiner, H.; Schalko, J.; Jachimowicz, A.; Keplinger, F. Accurate analytical model for air damping in lateral MEMS/MOEMS oscillators. Sens. Actuators A Phys. 2017, 255, 154-159, doi:10.1016/j.sna.2016.12.023.

(C) 2018 by the authors. Licensee MDPI, Basel, Switzerland. This article is an open access article distributed under the terms and conditions of the Creative Commons Attribution (CC BY) license (http://creativecommons.org/licenses/by/4.0/). 